\title{
Design of multilayered space filters using conductive film grid sheet and dielectric materials
}

\author{
Ryudai Ebara $^{1, \text { a) }}$, Shinichiro Yamamoto ${ }^{1, \text { b) }}$, Satoru Aikawa ${ }^{1}$, \\ Shigeki Matsuoka ${ }^{2}$, and Masaki Nagao ${ }^{2}$ \\ ${ }^{1}$ Graduate School of Engineering, University of Hyogo, \\ 2167 Shosha, Himeji, Hyogo, Japan \\ ${ }^{2}$ Zippertubing (JAPAN), Ltd., 3-2-56 Takatsukadai, Nishi-ku, Kobe, Hyogo, Japan \\ a)ei20d006@steng.u-hyogo.ac.jp \\ b)yamamoto@eng.u-hyogo.ac.jp
}

\begin{abstract}
As the newly EMC materials, the authors have been proposed the bandpass space filters consisting the metal wire grid with negative relative permittivity and dielectric materials. In this study, the novel space filters using conductive film grid, instead of a metal wire grid, are proposed. Next, the transmission characteristics of the proposed space filters for a Wifi router in the $2.4 \mathrm{GHz}$ band or the $5.0 \mathrm{GHz}$ band are evaluated by the measurement in free space and the calculation of transmission line theory.
\end{abstract}

Keywords: bandpass space filter, conductive film grid, dielectric material Classification: Electromagnetic Compatibility (EMC)

\section{References}

[1] B.A. Munk, Frequency Selective Surfaces-Theory and Design-, pp. 26-62, Wiley-Interscience Publication, 2000. DOI: 10.1002/0471723770

[2] A. Kazem Zadeh and A. Karlsson, "Capacitive circult method for fast and efficient design of wideband radar absorbers," IEEE Trans. Antennas Propag., vol. 57, no. 8, pp. 2307-2314, 2009. DOI: 10.1109/TAP.2009.2024490

[3] T. Nishiuchi, S. Yamamoto, K. Hatakeyama, T. Iwai, and S. Aikawa, "Design of frequency selective EM shielding materials using artificial designed materialsevaluation of transmission characteristics from microwave to millimeter-wave band-," IEICE Technical Report, EMCJ2019-14, pp. 7-12, 2019.

[4] T. Iwai, S. Yamamoto, and K. Hatakeyama, "Reflection and transmission characteristics of multilayered structure consisting of metal wire grid and dielectric layers," IEICE Trans. Commun. (Japanese Edition), vol. J95-B, no. 3, pp. 488492, 2012.

[5] M. Okita, S. Yamamoto, K. Hatakeyama, and T. Tsutaoka, "Band-pass space filters by laminating wire grids and high permittivity materials," IEICE Trans. Commun. (Japanese Edition), vol. J100-B, no. 3, pp. 205-209, 2017.

[6] K. Hatakeyama, T. Tsutaoka, T. Kanemoto, S. Yamamoto, and T. Iwai, "Reflection and transmission characteristics of EM-waves with the wire-grid and its use as a back layer of EM-wave absorber," IEICE Trans. Commun. (Japanese Edition), vol. J93-B, no. 1, pp. 101-111, 2010.

[7] N. Marcuvitz, Waveguide Handbook, pp. 285-289, McGraw-Hill, 1951. 
[8] Y. Sugiyama, T. Nishiuchi, S. Yamamoto, K. Hatakeyama, and K. Tada, “Transmission coefficient measurement setup for automotive millimeter-wave rader cover," IEICE Technical Report, EMCJ2017-84, pp. 47-52, 2017.

[9] S. Yamamoto, T. Iwai, and K. Hatakeyama, "Measurement setup of transmission coefficient for EM-wave shielding panels at microwave frequencies," IEICE Trans. Commun., vol. E94-B, no. 6, pp. 771-774, 2011.

\section{Introduction}

Recently, wireless communication systems, such as smartphones and 5G communication are rapidly increasing. On the other hand, the various issues such as interference of electromagnetic (EM) waves between the electronic equipment caused by undesired EM waves. To eliminate these undesired EM noises, EM wave absorbers and EM shielding materials consisting various metallic patterns have been reported $[1,2]$.

In our previous studies, EM shielding materials which made of artificial designed materials, for example, metal wire grid and conductive material, were designed [3]. In addition, as the application example, we have been proposed the multilayered space filters inserted metal wire grid between dielectric materials, and confirmed that the total transmitted frequency of proposed filters can be controlled by changing the structural parameters of the metal wire grid and the dielectric materials $[4,5]$.

In this study, the authors proposed the newly space filters that are thinner and lighter than the conventional structures. In the proposed space filter, the conductive film grid sheet was used instead of the metal wire grid. Next, the transmission characteristics were evaluated by measurement in free space and transmission line calculations. By these considerations, we confirmed that the frequency selective space filters for a Wifi router frequency band can be obtained.

\section{Proposed space filter consisiting conductive film grid sheet and dielectric materials}

\subsection{Conductive film grid sheet}

Figure 1 shows the construction and a photograph of the proposed conductive film grid sheet. We confirmed that the conductivity of this conductive film shows $1.24 \times 10^{5}[\mathrm{~S} / \mathrm{m}]$ by the measurement results of four-terminal method. The conductive film grid is made by arranging conductive cloth of constant width at constant interval. In this study, the width $d$ is fixed at $1 \mathrm{~mm}$ and the thickness $t$ is fixed at $0.04 \mathrm{~mm}$ because these values are lightest and thinnest to maintain structure, then the vertical and horizonal grid array intervals $a$ is changed to evaluate the transmission characteristics. The total structual size of the conductive film grid is a round shape with a diameter of $20 \mathrm{~cm}$.

The conductive film grid is expressed as the impedance $Z_{F}$ inserted in parallel with the transmission line [6]. $Z_{F}$ is represented as follows.

$$
Z_{F}=R_{i}+j\left(X_{i}+X_{F}\right)
$$

Where, $R_{i}$ and $X_{i}$ are the real and imaginary parts of $Z_{F}$ per unit length of conductive 


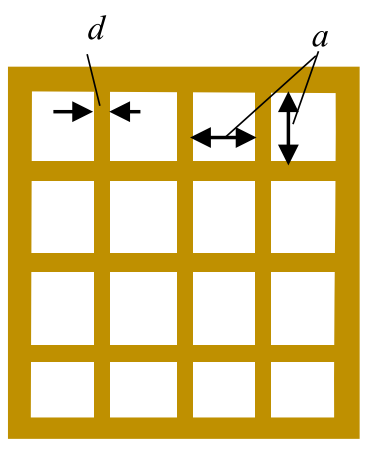

(a) Construction

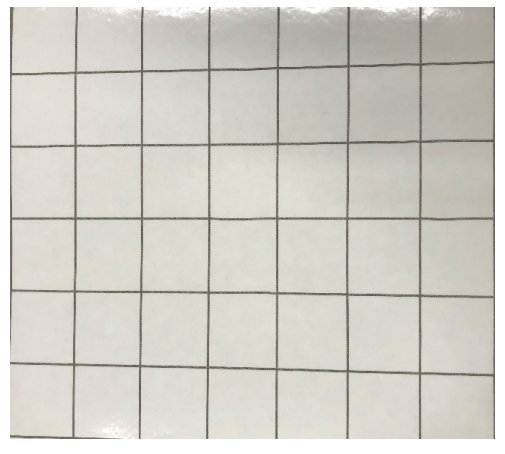

(b) Photograph

Fig. 1. Proposed conductive film grid sheet.

film grid. $X_{F}$ is reactance determined by conductive film grid with a resistivity of 0 , and it expressed by array interval $a$ of conductive film grid as follows.

$$
X_{F}=\frac{Z_{0} a}{\lambda_{0}}\left[\ln \left(\frac{a}{2 \pi r_{0}}\right)+0.601\left(\frac{a}{\lambda_{0}}\right)^{2}\right]
$$

Where, $Z_{0}$ and $\lambda_{0}$ are the impedance and the wavelength in free space, respectively. At this time, we regard a conductive film grid as a metallic foil because cross section of it is rectangle, so $r_{0}$ is as follows [7].

$$
r_{0}=\frac{d}{4}\left[1+\frac{d}{\pi t} \ln \left(4 \pi e \frac{t}{d}\right)\right]
$$

The relational expression of $R_{i} \ll X_{F}$ and $X_{i} \ll X_{F}$ is established when conductive film grid has a small enough resistance, so admittance $Y_{F}$ of conductive film grid inserted in parallel with the line is represented as follows [8].

$$
Y_{F}=\frac{1}{Z_{F}}=-j \frac{1}{X_{F}}=-j \frac{\lambda_{0}}{Z_{0} a\left[\ln \left(\frac{a}{2 \pi r_{0}}\right)+0.601\left(\frac{a}{\lambda_{0}}\right)^{2}\right]}
$$

\subsection{Newly proposed space filter consisting of conductive film grid sheet and dielectric materials}

In this study, we designed a newly space filter transmitting all of EM wave for Wifi in the $2.4 \mathrm{GHz}$ band or $5.0 \mathrm{GHz}$ band, which is one of the most popular wires system. Figures 2 (a) and 2 (b) show the construction of proposed space filter and the equivalent circuit based on transmission line theory, respectively. In this structure, we used acrylic plate (relative permittivity $\varepsilon_{r}: 2.85$, thickness $t: 2 \mathrm{~mm}$ ) as the dielectric material since we consider creativity. Also, we assumed that there were free space layer with half the thickness of a conductive film grid on both sides of a conductive film grid [8].

Here, the four-terminal matrix of the acrylic plate and the free space in Fig. 2 (b) are represented by the following transmission line theory equations.

$$
\left[\begin{array}{ll}
A_{r} & B_{r} \\
C_{r} & D_{r}
\end{array}\right]=\left[\begin{array}{cc}
\cosh \left(\gamma_{r} t_{r}\right) & Z_{r} \sinh \left(\gamma_{r} t_{r}\right) \\
\frac{1}{Z_{r}} \sinh \left(\gamma_{r} t_{r}\right) & \cosh \left(\gamma_{r} t_{r}\right)
\end{array}\right]
$$




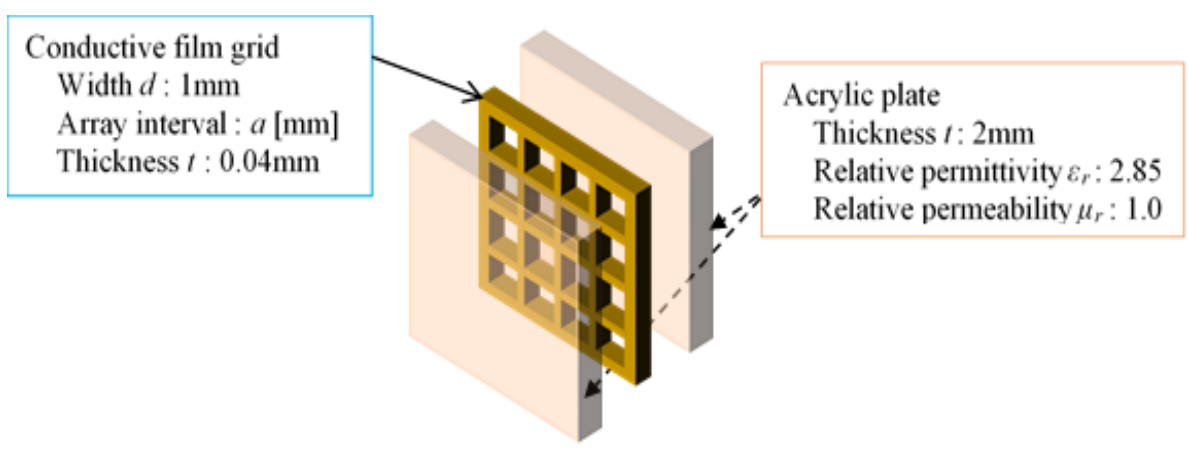

(a) Configuration of proposed space filter.

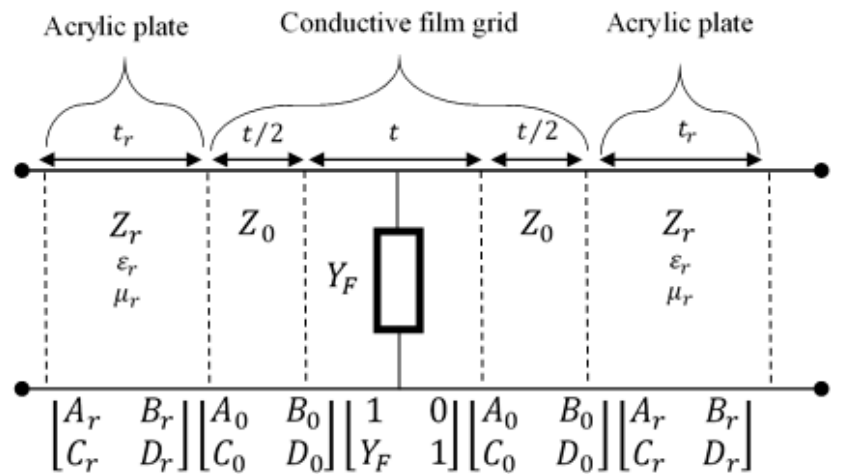

(b) Equivalent circuit of multilayered structure.

Fig. 2. Construction and equivalent circuit of proposed space filter.

$$
\left[\begin{array}{ll}
A_{0} & B_{0} \\
C_{0} & D_{0}
\end{array}\right]=\left[\begin{array}{cc}
\cosh \left(\gamma_{0} \frac{t}{2}\right) & Z_{0} \sinh \left(\gamma_{0} \frac{t}{2}\right) \\
\frac{1}{Z_{0}} \sinh \left(\gamma_{0} \frac{t}{2}\right) & \cosh \left(\gamma_{0} \frac{t}{2}\right)
\end{array}\right]
$$

Where, the propagation constants $\gamma_{r}$ and $\gamma_{0}$ of Eqs. (5) and (6) are expressed by

$$
\gamma_{r}=j \frac{2 \pi}{\lambda_{0}} \sqrt{\varepsilon_{r} \mu_{r}}, \quad \gamma_{0}=j \frac{2 \pi}{\lambda_{0}}
$$

and characteristic impedance $Z_{r}$ is expressed as follows.

$$
Z_{r}=Z_{0} \sqrt{\frac{\mu_{r}}{\varepsilon_{r}}}
$$

Besides, the four-terminal of whole laminated structure is calculated by cascading the four-terminal of each layer. The four-terminal of whole structure is expressed as follows.

$$
\left[\begin{array}{ll}
A & B \\
C & D
\end{array}\right]=\left[\begin{array}{ll}
A_{r} & B_{r} \\
C_{r} & D_{r}
\end{array}\right]\left[\begin{array}{cc}
A_{0} & B_{0} \\
C_{0} & D_{0}
\end{array}\right]\left[\begin{array}{cc}
1 & 0 \\
Y_{F} & 1
\end{array}\right]\left[\begin{array}{cc}
A_{0} & B_{0} \\
C_{0} & D_{0}
\end{array}\right]\left[\begin{array}{cc}
A_{r} & B_{r} \\
C_{r} & D_{r}
\end{array}\right]
$$

The transmission coefficient $T$ can be calculated by following equation by using Eq. (9) [8].

$$
T=\frac{2}{A+B / Z_{0}+C Z_{0}+D}
$$




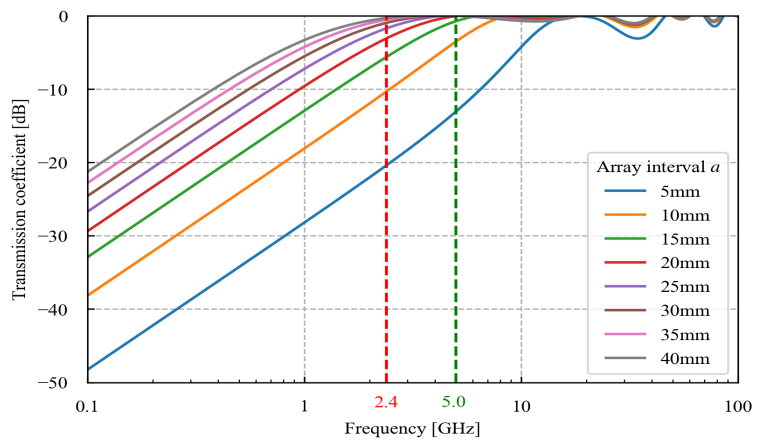

(a) Relationship between grid array interval and transmission coefficient.

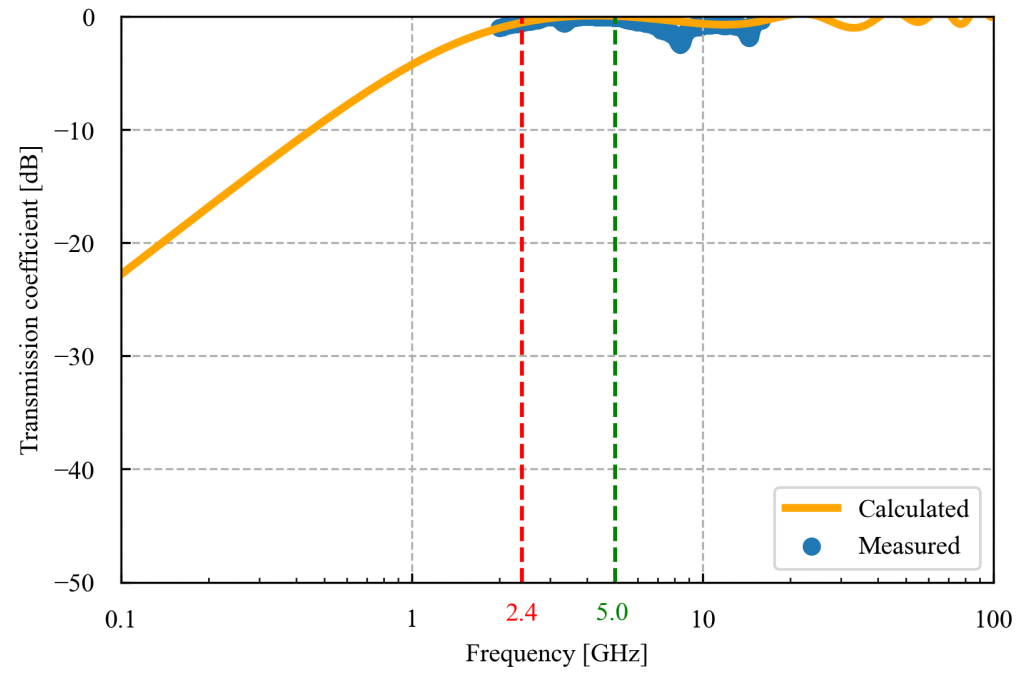

(b) Space filter total transmitting at $2.4 \mathrm{GHz}$ in the case of $a=35 \mathrm{~mm}$.

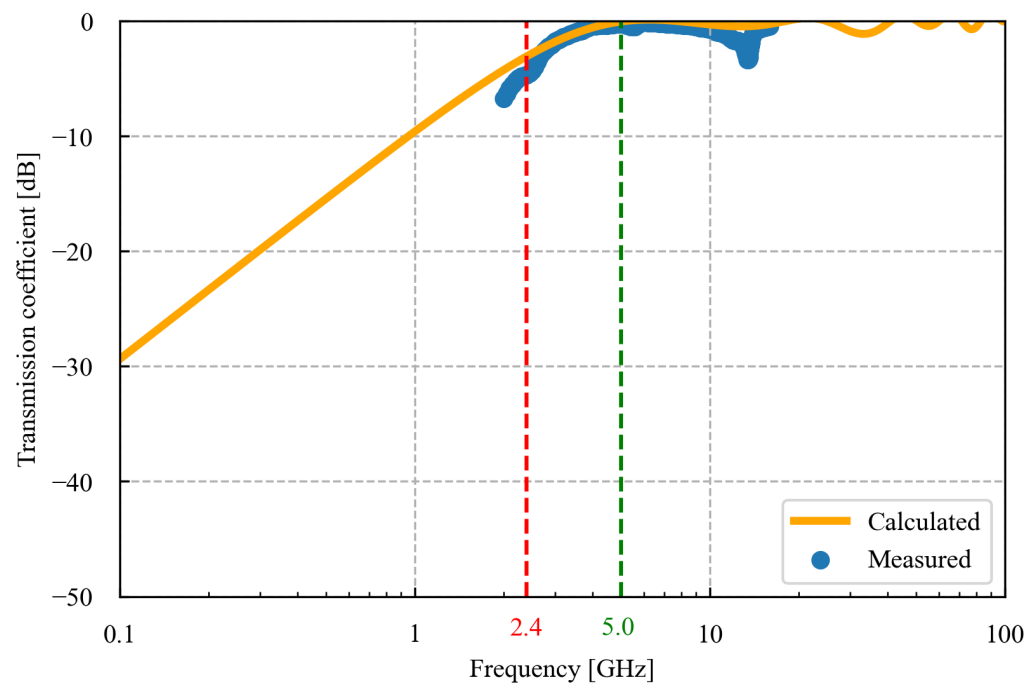

(c) Space filter total transmitting at $5.0 \mathrm{GHz}$ in the case of $a=20 \mathrm{~mm}$.

Fig. 3. Measured and calculated transmission coefficients of proposed space filters. 


\section{Transmission characteristics of proposed space filter}

In order to measure the transmission coefficient of the samples, the free space measurement setup [9] was used here. The transmission coefficient $T$ was obtained by Eq. (11). $T_{1}$ is the receiving power in the case with the sample, $T_{0}$ is the receiving power in the case without the sample, respectively. The dynamic range of the measurement setup is almost $-60 \mathrm{~dB}$ [9], and the measurement frequency range is from $2 \mathrm{GHz}$ to $16 \mathrm{GHz}$.

$$
|T|[\mathrm{dB}]=\left|T_{1}\right|-\left|T_{0}\right|
$$

Figure 3 (a) shows the calculated transmission coefficients by the transmission line theory when the grid array interval $a$ was changed from $5 \mathrm{~mm}$ to $40 \mathrm{~mm}$. From these results, we confirmed that the $a$ is smaller, the transmission coefficient becomes small. In addition, the total transmission characteristics $(|T|=0 \mathrm{~dB})$ can be achieved at $2.4 \mathrm{GHz}$ and $5.0 \mathrm{GHz}$ in the case of $a=35 \mathrm{~mm}, 20 \mathrm{~mm}$, respectively. Where, we didn't use array interval $a=40 \mathrm{~mm}$ because only four grid lines can be placed in the round shape with a diameter of $20 \mathrm{~cm}$, which is the sample size.

Figures 3 (b) and 3 (c) show the measured transmission coefficient of proposed space filter with the grid array interval $a=35 \mathrm{~mm}$ and $a=20 \mathrm{~mm}$. Also, the calculated results by the transmission line theory are shown in the same figure. From these results, there is a good agreement between calculated and measured values. Also, then the transmission coefficient becomes small with the frequency decreases. Moreover, we confirmed that this structure operates as a bandpass space filter because it is approximately $0 \mathrm{~dB}$ almost at $2.4 \mathrm{GHz}$. Besides, in Fig. 3 (c), calculated and measured values show in good agreement as well as in Fig. 3 (b). We confirmed that this structure operates as a bandpass space filter because it is nearly $0 \mathrm{~dB}$ almost at $5.0 \mathrm{GHz}$.

By above considerations, we found that it is possible to design multilayered space filter using the conductive film grid sheet. In order to design a sharp bandpass space filter, it is necessary to use a dielectric material with higher relative permittivity.

\section{Conclusion}

In this study, we designed the newly space filters consisting a conductive film grid sheet and the dielectric materials. By evaluating the measured and calculated transmission characteristics, we confirmed that the frequency selective space filter can be achieves at $2.4 \mathrm{GHz}$ and $5.0 \mathrm{GHz}$ for Wifi frequency band.

As the future works, there are designing steeper bandpass space filter used a higher relative permittivity dielectric material and a highpass space filter with structure in which conductive film grid are arranged on both sides of dielectric materials. 